\title{
What could be My Next Job? Using Flat Information Structures to Generate Creative Future Career Ideas
}

\begin{abstract}
In a dynamic labor market, it is important to help people combine information and generate creative solutions to cope with complex career challenges. In the present research, we apply the theory of information structure to creative career idea generation and hypothesize that flat information structures - that is, structures in which the information is disorganized are more conducive to creativity than hierarchical me mical information struture that is, strutures in which inform two experimental studies, participants had to combine career information related to self and work preferences that was presented either in flat or hierarchical structures. We found that flat information structures, compared with hierarchical information structures, led to future career ideas that were more creative on average. Our results suggest that cognitive flexibility explains the effect of information structure on the creativity of career ideas. Theoretical implications and suggestions for career management practices are discussed.
\end{abstract}

Keywords: information structure, creativity, career decision-making, cognitive flexibility.

In today's world of work, we are experiencing constant change and increased complexity (Baruch, 2004; Savickas et al., 2009). Individuals no longer have long-term, linear careers, but instead make a series of career changes throughout their lives (Savickas et al., 2009). Researchers have suggested that creativity-the capacity to produce original and appropriate ideas (Amabile, 1996) - is critical for individuals undergoing such career changes (Peiperl, Arthur, Goffee, \& Anand, 2002). Indeed, career adaptation often requires individuals to reflect on personal career information and to generate new career solutions (Watson \& McMahon, 2018).

Recent fundamental research in the field of creativity suggests that information structures that are flat (or unstructured), as opposed to hierarchical (or structured), increase flexibility in the cognitive processing of this information and thereby facilitate the production of creative ideas based on this information. In contrast, hierarchical information structures impose mental categorization and therefore lead to less creative idea generation in comparison (Kim \& Zhong, 2017).

In the present work, we investigate whether the presumed flexibility in idea generation as a function of information structure could be applied to the career domain (Runco \& Chand, 1995). In two experimental studies, we aim at testing (a) whether information structure can impact the creativity of future career ideas generated by workers or by undergraduate students and (b) whether cognitive flexibility could explain the effect of information structure on the creativity of future career ideas.

\section{THE INCREASING ROLE OF CREATIVITY IN CAREER MANAGEMENT}

Traditionally, career paths were predictable and stable. The typical individual learned a specific skill set, chose an occupation, and remained loyal to one organization over their entire working life (Baruch, 2004; Hall, 2004; Savickas et al., 2009). Factors such as globalization, technological innovation, and the dynamic 